\title{
Twin Earth and perceptual content
}

\author{
Jan Almäng ${ }^{1}$ (B) \\ Received: 14 March 2019 / Accepted: 24 October 2019 / Published online: 1 November 2019 \\ (c) The Author(s) 2019
}

\begin{abstract}
This paper presents a framework for analysing perceptual Twin Earth thought experiments. Visual content normally has an analogue character, and it is argued in this paper that this sets certain constraints on the extent to which Twin Earth thought experiments can be successful. The argumentation in the paper is developed by using examples from visual spatial content. It is argued that visual spatial content can only be "twin-earthed" in a very limited way. Whereas the metrics of space can be twinearthed, visual experience has a structure that means that it can only be the vehicle for representing entities with geometrical structures.
\end{abstract}

Keywords Twin Earth $\cdot$ Space $\cdot$ Perception

\section{Introduction}

Ever since the publication of Hilary Putnam's seminal paper "Meaning and Reference" (Putnam 1973) has the scope and nature of content-externalism been a popular subject in philosophy of mind. Putnam showed that two persons who were intrinsically alike could nevertheless mean different things by the same word. There has been no shortage of philosophers who have attempted to develop Putnam's basic idea. In this paper we shall focus on attempts to develop the idea with respect to perceptual content. More specifically, the paper will focus on theories according to which two "phenomenal twins" could perceptually represent different types of entities.

The main question that is raised in this paper is to what extent the phenomenal character of a perceptual experience determines the perceptual content of the perceptual experience, where the phenomenal character of the experience is conceived of as the instantiation of phenomenal (and not the represented) properties in the experience. The main contention in the paper is that whereas there are some kinds of perceptual content that are amenable to Twin Earth thought experiments, the nature of perceptual

\footnotetext{
Jan Almäng

Jan.almang@gmail.com

1 Faculty of Arts and Social Sciences, Karlstads universitet, $65188 \mathrm{Karlstad}$, Sweden
} 
experiences places limits on the extent to which perceptions can be "twin-earthed". I shall attempt to introduce a framework which describes these limits and apply it to the case of visual spatial content. I will suggest that the geometrical structure of visual experience means that spatial content can only be "twin-earthed" in a very limited sense. In the concluding section of the paper I shall suggest that this gives us some reason to believe that spatial content is to some extent internally individuated. ${ }^{1}$

My paper will —in a broad sense-be located within the framework provided by phenomenal intentionalism. ${ }^{2}$ I will assume that the phenomenal character of a visual experience has an important explanatory role with respect to the intentional content of the said experience and that perceptual Twin Earth cases are characterised by the fact that "twins" have experiences whose phenomenal character are qualitatively identical. If we assume that perceptual experiences instantiate phenomenal properties, phenomenal twins will have experiences which instantiate the same types of phenomenal properties. I will moreover assume that the perceptual vehicles of representation are phenomenal entities. So on this conception, a successful Twin Earth case is one where a phenomenal property (or other type of entity) is the vehicle for representing one type of entity on Earth, but a quite different type of entity on Twin Earth.

I will consequently assume that the phenomenal character of perceptual experience does not supervene on the intentional content of the said experience. In my opinion there are good reasons for rejecting this kind of representationalism since this view of the relationship between phenomenal character and representational content seems to preclude that two individuals could be in the same phenomenal state but different representational states. But it lies beyond the scope of this paper to defend this view here. $^{3}$

Sections two to four set the stage for the argumentation in the paper. The second section of the paper presents the notion of analogue perceptual content. The third section describes the nature of Twin Earth thought experiments and what would constitute a successful Twin Earth scenario. My main contention here is that a successful Twin Earth thought experiment with respect to perceptual content is one where each vehicle of representation on Earth, is also a vehicle of representation on Twin Earth. If this is not the case, the scenario fails.

Section 4 of the paper presents the notion of phenomenal space and how it is the vehicle for representing geometrical structure in our world. Phenomenal space is a notion that I borrow from Dainton (2000) and it will be of crucial importance in this paper. I will following Dainton argue that we should conceive of the phenomenal

\footnotetext{
${ }^{1}$ My claim is not entirely original. Horgan, Tienson and Graham have suggested something similar, even though their argumentation is quite different (or even absent in some respects) than mine. They claim that reference to spatiotemporal locations, shapes and sizes are constituted by phenomenology alone, regardless of whether or not the experiencing subject is a normal human being or a brain in a vat (Horgan et al. 2004: p. 316, cf Horgan and Graham 2012: p. 335). I disagree with respect to sizes and agree with respect to locations and shapes. Shapes are by and large absent from this paper; for an argument to the effect that they are not twin-earthable, see Almäng (2017).

2 The literature on phenomenal intentionalism is vast. For some of the most important work, see for example Chalmers (2006), Farkas (2008), Horgan et al. (2004), Horgan and Graham (2012), Kriegel (2011, 2012), Siegel (2010), Siewert (1998) and Smith (2002).

${ }^{3}$ For a classical argument along these lines against representationalism, see Block (1990). For a defence of representationalism that attempts to meet Block's challenge, see Tye (1998).
} 
character of visual experience as having a spatial character and that phenomenal space is distinct from physical space. Section 4 concludes by noting that phenomenal space has four important characteristics. It has three dimensions. It consists of a set of points. Each point or region of points can be either occupied or empty. And each pair of points is related by a distance.

Sections 5-8 shows how each of these characteristics set certain constraints on the extent to which spatial content can be the vehicle for representing other entities than they do on Earth. Section 5 argues that our visual experiences can only be the vehicles for representing a three-dimensional space. Section 6 argues that the ratio between the distances in phenomenal space must correspond to the ratio between the represented distances. Section 7 argues that the positions in our visual experience can only be the vehicle for representing positions in a space. Section 8 argues that the occupation-relation in our visual experience can only be the vehicle for representing that a position in space is occupied by some entity.

Section 9 concludes the paper and suggests that the structure of a visual experience is such that it can only represent a spatial structure that is isomorphic to the geometrical structure of phenomenal space. Phenomenal space cannot represent non-spatial entities. While a direct argument for the notion that phenomenal space is intrinsically intentional lies beyond this paper, I utilize Chalmers' notion of Edenic content in order to show that if we accept that perception has an Edenic content, phenomenal space is a kind of Edenic content that can be satisfied and that reveals the world as it is.

\section{Perception and analogue content}

Perception is commonly held to be different than thought in that it represents in a quite different way. This is sometimes expressed in terms of perceptions representing in an "analogue" way (cf Beck 2012) and that is the terminology we shall adopt in this paper as well. Following Brian Cantwell Smith (1991: p. 271) we shall say that a representation is analogue if the structure of the representation corresponds to the structure of what is represented (cf Smith 1991: p. 271). Analogue representations are in other words structurally isomorphic with the object of representation.

I will not attempt to give a precise definition of what an analogue representation is. Let us however emphasize three characteristics of analogue representations. These characteristics are not necessary conditions for a representation to be analogue. They are rather to be seen as three different ways that a representation can be structurally isomorphic with respect to what is represented and consequently qualify as an "analogue representation" in the sense of the term used here. The claim that visual experiences are characterised by analogue representations should be understood against the background of the notion of phenomenal space, that is developed in the fourth section. Given that phenomenal space has the characteristics attributed to it, visual experiences represent analogically in the sense described in this section.

The first characteristic is that $n$-dimensional entities are represented by $n$ dimensional vehicles of representation. The second is that properties or relations with a certain magnitude are represented by vehicles that have an analogous magnitude. The third characteristic is that relations between vehicles of representation can represent 
relations between the represented objects. Let us take a look at these three conditions in turn.

The first characteristic is that n-dimensional entities are represented by entities which themselves have $n$-dimensions. Let us for example consider space. It has been argued (cf Dainton 2000) that visual experiences are themselves three-dimensional. Dainton (2000) uses the term "phenomenal space" when describing the geometrical structure of visual experience, and we shall follow his usage in this paper. Phenomenal space functions as the vehicle for representing space on Earth. I shall also argue (in Sect. 4) that it is best conceived of as a kind of space itself-it has the same geometric structure as physical space.

On the conception used in this paper, the vehicles for perceptually representing various entities such as properties, relations and objects are phenomenological entities. We shall call the vehicles for representing colours in our world "phenomenal colours", the vehicle for representing blue in our world "phenomenal blue", the vehicles for representing shapes in our world "phenomenal shapes", and so on and so forth.

Space is not the only perceivable entity that is multidimensional. Funkhouser (2014) analyses properties in terms of non-determinable necessities and property-spaces. A non-determinable necessity is a structural feature that all properties of a given determinable have by necessity. Beliefs for example, are by necessity characterised by the feature "holding true". They do not vary in this respect. However, beliefs can vary with respect to what is believed (their propositional content) and the degree of confidence that is assigned to the belief. (Funkhouser 2014: p. 36) Properties have according to Funkhouser values along multiple dimensions. For beliefs, the dimensions are degree of confidence and propositional content. Together, these can symbolically be represented by a two-dimensional property space. Properties with more than two dimensions obviously form property-spaces with more than two dimensions. Different points in a property space represent properties with different values along their determination dimensions but with the same non-determinable necessities.

Let us assume that the analysis of colour in terms of hue, saturation and lightness is correct. It then follows that colours are three-dimensional properties. They can differ with respect to three dimensions. We may then conjecture that phenomenal colours - the vehicle for representing colours, have values in three-dimensions.

We can obviously perceive one-dimensional properties and relations as well. Force is for example a one-dimensional property and the perception of force by means of our tactile senses is presumably represented by a one-dimensional vehicle-phenomenal force. Distance is a one-dimensional relation between locations in space. And it is once again represented by a one-dimensional vehicle_-phenomenal distance. Phenomenal distances are however located in three-dimensional phenomenal space and unlike phenomenal forces they are not properties but relations.

Our second characteristic is that properties or relations with a certain magnitude are represented by vehicles with a certain magnitude. If we represent one magnitude as larger than another, then the vehicle for representing the first magnitude must be of a larger magnitude than the vehicle for representing the second magnitude. As Jacob Beck puts it when outlining his argument to the effect that perception is analogue, the claim is that "the mind represents objective magnitudes by way of mental magnitudes". (Beck 2019: p. 329, cf Beck 2012: p. 589) 
If we represent a force, then we may assume that not only the force but also the vehicle for representing the force-the phenomenal force-has a certain magnitude. The larger the force that we represent, the larger is the phenomenal force that is the vehicle for representing the force. It is important to note that the represented magnitude and the vehicle that is representing the magnitude need not be magnitudes of the same kind. Whatever the magnitude of phenomenal force is, it is presumably not a force itself.

Our third characteristic is that relations between vehicles of representation can represent relations between what is represented. They can in other words themselves be vehicles of representation. Consider for example the perception of spatial distance. It is reasonable to assume that a prerequisite for perceiving a distance between two locations in space is that we perceive these locations and that the vehicle for perceiving a position is itself a location (or position) in the three-dimensional visual experience. And the vehicle for representing the distance between the represented locations, is itself a relation-phenomenal distance - that holds between the locations in phenomenal space.

Or consider the perception of magnitudes. It is not merely the case that we can perceive magnitudes. We can also perceive relations between magnitudes. Thus, for example, we can perceive one distance as being twice as long as another distance, or one object as twice as heavy as another object. In this case it is reasonable to assume that the perception of the relation (twice as long or twice as heavy) is represented by the relation that holds between the vehicles representing distances and forces. So, for example, we represent one object as twice as heavy as another object because the phenomenal weight representing one object bears a ratio of $2: 1$ to the phenomenal weight that is representing the lighter object.

It is especially noteworthy that when we visually represent mereological relations such as parthood, then the vehicles for representing these relations are themselves isomorphic with respect to what is represented. The vehicle for representing a brick may for example be a part of the vehicle for representing the wall that the brick may be represented to be a part of.

But mereological isomorphism is not merely something that pertains to the perceptual representation of material objects. The principle applies also with respect to the perception of distances. If you perceive a path in space between points $\mathrm{A}$ and $\mathrm{B}$, where point $\mathrm{B}$ lies in the path between $\mathrm{A}$ and $\mathrm{C}$, then the vehicles for perceiving the paths $\mathrm{AB}$ and $\mathrm{BC}$, are parts of the vehicle for perceiving $\mathrm{AC}$.

This completes my presentation of the notion of analogue content. We shall call our three characteristics dimensionality, magnitude and relationality.

\section{Twin Earth thought experiments}

Putnam describes his Twin Earth scenario in terms of two persons being physical duplicates of each other, but nevertheless representing different entities with their words. In this paper I shall use a slightly different way of formulating Twin Earth scenarios, one that can be found in the works of Farkas (2003, 2008 and cf Burge 2010: p. 76 for a similar, but not identical conception). She (2003: p. 196f) emphasizes that inten- 
tional content is external if two individuals could have subjectively indistinguishable experiences yet have different contents.

This broadly phenomenological way of framing the difference between internalism and externalism is the one I will use in this paper. A Twin Earth scenario is on this conception a scenario where a pair of phenomenal twins represent different types of entities. This is important to emphasize. Most philosophers would agree that phenomenal twins that are situated in different environments represent different particulars. The crucial question is if, and if so to what extent, phenomenal twins could also represent different types of entities. ${ }^{4}$

Twin Earth scenarios could be successful with respect to one type of entities, but fail with respect to another type of entities. Thus, for example, there is nothing that prevents Twin Earth scenarios from being successful with respect to colours (cf Block 1990), but unsuccessful with respect to shapes. If this were the case, it is possible that phenomenal twins could represent different colours, but impossible that they could represent different shapes.

Twin Earth scenarios are normally taken to give support to externalism with respect to intentional content. The idea is that if a vehicle of representation can reliably track different types of entities in different contexts, then the vehicle of representation is not intrinsically representing anything. It is however important to note that if it can be shown that there is no Twin Earth scenario for a vehicle of representation, we have only shown that the vehicle in question cannot reliably track more than one type of entity. We have not shown that the vehicle is intrinsically intentional. The reason is that we may conceive of scenarios where the vehicle is not reliably tracking anything. And it is possible for the externalist to claim that in such cases the vehicle is not representing anything.

I shall assume that in order for a Twin Earth scenario to be successful, it must be shown that a vehicle could reliably track different types of entities. Two entities are different types in the sense used here if they are not qualitatively identical to each other. Thus, what makes it possible for phenomenal blue to represent blue in some contexts and green in other contexts is that phenomenal blue can reliably track both blue and green. So if it can be shown that a vehicle for representing something on Earth cannot reliably track other entities than it does on Earth then it is on the conception used here not a twin-earthable vehicle.

Reliable tracking is a necessary condition for a Twin Earth scenario to be successful, but not a sufficient condition. A different condition that must be fulfilled is that the type of entity that is tracked by a vehicle of representation is a "natural" entity, and not

\footnotetext{
4 It is to be noted that this formulation of Twin Earth scenarios presupposes that the phenomenal character of a perceptual experience is not constituted by or identical to the objects and properties perceived. According to some representationalists, the phenomenal character of a perceptual experience is best conceived of as a Russellian proposition that has the objects and properties that the experience represents as constituents (cf Tye 2002). In a similar vein, disjunctivists (cf Martin 2004; Snowdon 2005) normally claims that the perceived objects and properties are part of the phenomenal character of a perceptual experience. However, it is hard to see how the kinds of Twin Earth scenarios that are discussed in this paper could even be conceivable if this was correct, so I shall simply assume that the phenomenal character of an experience is not constituted by the objects of the experience or explained in terms of a Russellian proposition that contains the objects and properties that are represented in the experience.
} 
a wildly disjunctive or gerrymandered entity. Unless this condition is fulfilled, more or less any Twin Earth scenario will turn out to be successful. ${ }^{5}$

A Twin Earth scenario is successful if it can be shown that any phenomenal entity that functions as a vehicle of representation on Earth, also functions as a vehicle of representation on our Twin Earth. If a phenomenal entity functions as a vehicle of representation on Earth but not on Twin Earth however, then the scenario is unsuccessful. We shall say that a Twin Earth scenario is successful if it has been shown that our phenomenal twins are not representationally impoverished in comparison to us.

The representational content of the experiences of our phenomenal twins must in other words be as rich as our experiences given some tracking account of intentionality. ${ }^{6}$ So if a Twin Earth scenario shows that two vehicles of representation could reliably track different entities than they do on Earth, but that the relation that holds between them and that is a vehicle of representation on Earth cannot reliably track a relation in that scenario, then this scenario is not a successful scenario. Representationally impoverished scenarios are unsuccessful scenarios because in those cases it has not been established that all vehicles of representation on Earth might reliably track different entities in this scenario than they do on Earth. So if vehicles of representing relations are not reliably tracking anything in a Twin-Earth scenario, than it has not been shown that they are twin-earthable.

Before we conclude this section it is important to point out that the fact that brainsin-vats could be our phenomenal twins does not show that the brain in vat scenario is a successful Twin Earth scenario. David Chalmers has suggested that a brain in a vat would have perceptual experiences which are caused by computational properties and relations. Consequently, "our spatiotemporal expressions will pick out the computational properties and relations that serve as their causal basis, and those properties and relations really are present in the computer." (Chalmers 2012: p. 335)

Chalmers does not explicitly claim that the structure of visual experience would in this case represent something other than space, but it is clearly easy to see how his argument could be extended to that effect. It is however important to note that the fact that it is possible for us to have phenomenal twins who are brains in vats would not by itself show that a Twin Earth scenario of some kind is successful.

The fact that computational properties functions as the causal ground for our phenomenal twins does not entail that their experiences are reliably tracking computational properties. A could be the cause of $\mathrm{E}$, even though $\mathrm{E}$ does not reliably track A. For E might just as often be caused by B, C and D. And in this case, E would not reliably track anything. So the fact that we might have phenomenal twins who are brains in vats, where their experiences are caused by computational properties, does not entail

\footnotetext{
5 This is a version of the problem raised by Newman (1928). He noted that a theory that only represented the structure of something, would be true in any world with the right cardinality. Following Chalmers (2018) and Lewis (1984) my preferred solution to the problem raised is to limit the class of possible referents to natural properties and relations that, in a sense, "carve nature at its joints". It might be suggested with Lewis that naturalness comes in degrees and that we can refer to entities like grue, which are not entirely natural. That may well be the case, if so, I would have to retreat to the position that a successful Twin Earth scenario is one in which our phenomenal twins do not represent entities which are less natural and more gerrymandered than we do.

6 An internalist who reject tracking accounts of intentionality could obviously say that phenomenal twins always have the same representational content regardless of what, if anything, their experiences are tracking.
} 
the fact that these experiences reliably track any computational properties. Since I will argue that the geometrical structure of visual experience is not twin-earthable, it is important to bear in mind that this has no bearing on whether or not brain-in-vat scenarios are possible. (See also Sect. 8 in this paper for a description of a possibly successful Twin Earth scenario involving brains in vats.)

\section{Phenomenal space}

In this section I wish to suggest that our visual experience constitute a phenomenal space which is in our world the vehicle for representing physical space. The term "phenomenal space" was to my knowledge first introduced by Dainton (2000) but similar notions have been defended by other philosophers as well (cf Peacocke 1983).

In this section I shall present four assumptions concerning the nature of phenomenal space. My four assumptions are substantial, but not really original. Most of them have been defended in at least some version in the literature. Defending these assumptions in detail would require four separate papers. Here I shall mainly give a brief presentation of the assumptions and why I think that they are reasonable even though some of them are controversial.

We have already encountered the first assumption. Visual experience has a three-dimensional structure. The three-dimensional structure of visual experience is moreover in our world the vehicle for representing three-dimensional physical space (cf Dainton 2000, ch 3 for a long defence of this assumption).

The second assumption is that phenomenal space is like all other spaces populated by points. It is a set of points in a three-dimensional structure. These points are the relata of distance-relations, such that every pair of points in the set is related by a distancerelation. For human beings on Earth, points in phenomenal space functions as the vehicle for representing points in physical space, and regions of points in phenomenal space functions as the vehicle for representing regions of points in physical space.

I shall throughout this paper assume that points lack extension in all dimension. Nothing of any importance however hinges on this. Points can obviously compose larger regions and these are presumably of greater phenomenological importance than the points that constitute them. If someone were to object that we cannot discern non-extended points in phenomenal space, but merely regions that were extended, the account could be reformulated in terms of phenomenal space being populated by positions which are spatially extended.

The third assumption is that we can perceive space not merely as occupied, but also as empty. As Michael Martin has pointed out, when we perceive ring-shaped entities, we perceive not only the ring itself, but the empty space "inside" the ring (Martin 1993: p. 214, for other defences of the same idea, see Mac Cumhaill 2015; Richardson 2009; Soteriou 2011). If we assume that we can perceive empty space, then we can obviously also perceive space as occupied by entities.

We may conceive of points in phenomenal space as positions in a structure. These positions may be occupied by a vehicle that represents entities of various kinds. But they may also remain unoccupied, as when we perceive two objects which are separated by empty space. The emptiness in phenomenal space is in this case the vehicle for 
representing the empty space between our two represented objects. When a position is occupied by a vehicle for representing something, we shall say that the vehicle is located at the position. ${ }^{7}$

It should be noted that on some accounts, for example Husserl's, we are in addition to perceiving the surface of a visible object also perceiving its rear side, albeit in a nonsensory manner. Husserl calls the surface that is presented in a sensory manner for the "abschattung" or adumbration of the object, while the rear side is presented by parts of the content that he describes as "empty intentions" (cf Husserl 1966: p. 3ff; cf Smith and McIntyre 1982: ch 5) On the account defended here, it is only the adumbration of the object that is part of phenomenal space. If we perceive an entity that, as it were, blocks us from seeing further, then phenomenal space will only contain vehicles for representing the front-surface of that object. Phenomenal space lacks vehicles for representing the rear side or inside of the object of perception.

My fourth assumption is that the vehicles for representing distancerelations - phenomenal distances — are relations between points in phenomenal space. Each of these relations has a certain magnitude. The magnitude of the represented distance between two points depends on the magnitude of the distance between the two representing points in phenomenal space.

There is an obvious objection to this claim. If you visually represent that two sticks have the same size, but where one stick is represented as being further away from you than the other, then there is a sense in which the vehicle for representing the second stick has a greater "extensity" in the "visual field" than the first stick. Does not this show that the vehicle for representing distance cannot be equated with distance in phenomenal space?

Actually, it does not show this. The argument only goes through if we equate phenomenal distances with "extensity" in a two-dimensional visual field. But to do so would be to ignore the fact that phenomenal space has three dimensions, and not merely two. This is not the place to discuss the problem of perspectival properties in perceptual content. For present purposes I shall simply assume that visual experience is three-dimensional. Exactly how we should construe the difference between the sticks in the example above does not matter for present purposes as long as it is accepted that phenomenal space is three-dimensional with a metric. ${ }^{8}$

\footnotetext{
7 Dainton (2000: p. 72ff) discusses two possible cases of phenomenal space, one that contains voids and the other that does not contain voids. Dainton agrees that visual phenomenal space contains voids, but claims that they are literally nothing. He defends a relationalist account of visual phenomenal space. On my account, we can distinguish between the position in the spatial structure and the entity that occupies it. I am not sure whether relationalists would accept this notion. It is however clear that realists with respect to spatial structure would accept it (cf Nerlich 1994, 2013).

8 We could describe it as simply a case in which the sticks are located at different distances from us, and that the vehicles for representing the sticks consequently are located at different distances from the position representing our viewpoint in phenomenal space. If this is the case, there is no need to assume that there is any phenomenal entity that is bigger than another entity (cf Smith 2002: p. 172; Hopp 2013; Jagnow 2008, 2012). There are however other alternatives. Gibson (1950) suggests that we distinguish between three-dimensional and two-dimensional aspects of the experience, where the latter is an abstraction from the former. It is only in the latter in which the vehicles for representing the sticks differ from each other. Marr (1982) suggests that we distinguish between 2, 5 and 3-dimensional representations. The former is a 2-dimensional representation where different points in the representation are assigned (possibly different)
} 
Distance in phenomenal space is tantamount to the apparent distance between points in space. And as the stick exhibits perceptual constancy even though it is rotating in space, the distance between the endpoints of the vehicle for perceiving the stick is constant even when we represent it as rotating in front of us.

It is of course possible to deny that phenomenal distance is a relation with a magnitude. But this claim is simply false. If we experience a straight line, then the line can be cut in two separate pieces, each of which would have a lesser magnitude than the whole. If the distance between $\mathrm{A}$ and $\mathrm{C}$ is represented by the phenomenal distance $A_{p} C_{p}$, and where $B$ is a point between $A$ and $C$, then surely the distance $A B$ is represented by the phenomenal distance $A_{p} B_{p}$, where the phenomenal distance $A_{p} B_{p}$ can be described as shorter than the phenomenal distance $\mathrm{A}_{\mathrm{p}} \mathrm{C}_{\mathrm{p}} \cdot{ }^{9}$

I shall describe these four assumptions of phenomenal space as the structure of phenomenal space. I do not think that they exhaust the structure of phenomenal space. But these four assumptions are those that we will focus on. I shall suggest that each sets some constraints on what phenomenal space can represent.

But why, then, should we assume that there is such an entity as a phenomenal space? It is possible to defend the assumption in three steps. I do not have the space here to defend them at length, but it is possible to give a brief defence.

The first step is an assumption we have already encountered: The phenomenal character of a visual experience cannot be explained in terms of the objects of experience. It might of course be suggested that this assumption is false, and I would expect most Russellian Representationalists (i.e. those who hold that representational content is Russellian and that the phenomenal character of an experience supervenes on the representational content of the said experience) to reject it. But that is certainly not of any help to the friend of spatial twin-earthability who relies on the assumption in order to show that our experiences might have been representing some other entities.

The second step claims that the phenomenal character of our experience has a certain structure. In this respect it is similar to other mental states like, for example, thoughts. Indeed, it is similar to every complex entity. But just as it is possible to examine the structure of other mental states is it possible to examine the structure of the phenomenal character of our visual experiences. ${ }^{10}$

\section{Footnote 8 continued}

depths. On such an account, the 2.5 dimensional representations of the sticks would differ from each other, but not the 3-dimensional representations.

9 One of my referees has pointed out that physical space has a curvature and is non-Euclidean. Is phenomenal space Euclidean or not? If it is, it can only be type-identical to a local region of physical space since it is only smaller regions of physical space that can be described as roughly Euclidean in character. I have no firm answer to this question. I think we intuitively conceive of our experience of space as Euclidean but there are some reasons to believe that it actually could have a non-Euclidean character as well (cf Nerlich 1994: p. 38; Mac Cumhaill 2015 for such defences).

10 One of my referees points out that the second step is not uncontroversial given that perception may on some accounts be transparent. Consequently, it would be difficult to examine the structure of the phenomenal character under such circumstances. I agree that if experiences are transparent, then the second step becomes more controversial (It might still be possible to examine the phenomenal character in other ways than phenomenological ones though). However, I would like to point out that a Twin Earth scenario is here conceived of as one where two phenomenal twins could represent different entities. And if that is the case, the twins would be in different representational states but the same phenomenal state. So the kind of Twin Earth scenarios under discussion in this paper seems to preclude the possibility that perceptual experience is transparent in this sense. 
The third step claims that the structure of phenomenal space is type-identical to physical space in the sense that it has the four characteristics described above. Why should we assume that? The simple answer is that this is the best explanation for the phenomenal character of visual experience. Visual experience has a spatial phenomenology. And since by the first step of the argument we are unable to explain this phenomenology in terms of the represented physical space, the best explanation is one that assumes that the phenomenal character has a structure which is type-identical to physical space.

It is obviously possible to deny the third step, but the alternatives are not that attractive. Let us for example assume that the phenomenal character of a visual experience is not three-dimensional. Then how many dimensions does it have? It is certainly not one-dimensional. Perhaps it could be described as two-dimensional, but that does not explain the fact that visual experience seems to have a certain "depth".

Tyler Burge has claimed "that geometrical structures in sensory-motor memory correlate with the structure of physical space" (Burge 2010: p. 513) and that the "realized structure is mathematically equivalent to the structure described by a geometry that is representationally applied to [...] relations in physical space". (Burge 2010: p. 513) Burge, quite obviously, denies that these structures are intrinsically intentional. Nevertheless, the claim made with respect to phenomenal character should not be ontologically more controversial than the claims made by Burge. The main difference is that my claims pertain to phenomenal character whereas his claim pertains to the sensory-motor memory.

It is important to note that phenomenal space is not physical space. Facts about phenomenal space are presumably grounded by physical facts. But while phenomenal space might be grounded by facts pertaining to a region of physical space (such as a brain), phenomenal space is not a region of physical space. It is obviously not the case that there is empty physical space in the brain of a perceiver whose phenomenal space contains empty positions.

\section{Dimensional Twin Earth scenarios}

I have assumed that phenomenal space has three dimensions and it is obviously the case that it represents a three-dimensional world on Earth. But could we conceive of scenarios where phenomenal space represents entities with more or fewer dimensions than on Earth? Could we for example conceive of a case where phenomenal space represents a four-dimensional space or a two-dimensional space?

I shall argue that this is not possible. I shall in other words defend proposition (i) in this section.

(i) A phenomenal space with three-dimensions can only represent a space with threedimensions in a successful Twin Earth scenario.

As we shall see, (i) can easily be transformed into a proposition that is true not merely of phenomenal space but also of phenomenal properties that are vehicles of representations. 
Let us begin by pondering whether or not phenomenal space could represent a fourdimensional space. It is prima facie difficult to see how it could. Entities that are located in a four-dimensional space bear relations to each other that cannot be represented in a three-dimensional space. This is not to deny that phenomenal space could represent a three-dimensional segment of the four-dimensional world. That, however, would be tantamount to claiming that phenomenal space could represent a three-dimensional space, but this is obviously something that we already knew.

Nor is this to deny that we could represent a four-dimensional space in some other way. A two-dimensional map can for example represent a three-dimensional space by letting colours represent depth in the third dimension, and we could in an analogous way conceive of how phenomenal colours might represent a fourth spatial dimension. But a three-dimensional structure on its own cannot represent a four-dimensional structure. The relations between the points in a two-dimensional map cannot themselves be the vehicles for representing distance in a three-dimensional space. Something similar would be true if we assumed that phenomenal colours were representing depth in a fourth spatial dimension. Distances in three-dimensional phenomenal space would in such a case not represent any distances in four-dimensional physical space.

Could phenomenal space represent a two-dimensional space? This is certainly possible but a phenomenal twin of ours who represented a two-dimensional space would have an impoverished content in comparison to us. If phenomenal space represented a two-dimensional world, then one of the dimensions in phenomenal space would not represent anything. It would only be a two-dimensional segment of phenomenal space that represented anything. While this scenario is possible, it does not give us a successful example of a Twin Earth scenario.

The thesis that $\mathrm{n}$-dimensional entities can only reliably track other $\mathrm{n}$-dimensional entities is true not merely of phenomenal space but also of phenomenal vehicles that represent multidimensional properties. If a phenomenal vehicle with three determination dimensions is supposed to represent a one-dimensional property it would merely be one determination-dimension that was doing the representing. The others would not represent anything. Our phenomenal twins in this world would have an impoverished representational content in comparison to us.

\section{Metrical Twin Earth scenarios}

In this section we shall ponder to what extent the metrics of a space is twin-earthable. I shall suggest that our phenomenal twins must represent distances whose ratios are the same as on Earth.

(ii) The ratio between distances in phenomenal space can only represent ratios with which they are type-identical.

There is nothing that prevents our phenomenal twins from representing distances that are twice as long as distances on Earth as long as the ratio between the represented distances are the same as on Earth. 
An example of a successful Twin Earth scenario is Thompson's (2010) Doubled Earth. Doubled Earth is similar to Earth in all respects except that all distances are twice as large on Doubled Earth as on Earth. We have counterparts on Doubled Earth who are our phenomenal twins. However, their experiences reliably track distances which are twice as long as our distances. So, for example, if I perceive a stick that is $1 \mathrm{~m}$ long, my phenomenal twin on Doubled Earth perceives a stick that is $2 \mathrm{~m}$ long, and so on and so forth.

Is Doubled Earth a conceivable scenario? I think it is, and it nicely illustrates why spatial metrics can be twin-earthed. Phenomenal distances have a magnitude in phenomenal space and the length of the distance represented is a function of the magnitude of the phenomenal distance. Doubled Earth is not different from Earth in this respect. Our phenomenal twins obviously represent different distances than we do, but they nevertheless represent magnitudes by magnitudes.

According to relationality, relations between vehicles of representation can reliably track relations between entities in the world. It is obviously the case that Doubled Earth is similar to Earth in this respect. Any two objects that are spatially related on Earth have type-identical twins on Doubled Earth which are spatially related. And if one distance $A$ is represented as being twice as long as another distance $B$ on Earth by a perceiver, then the counterpart of $A$ is represented as being twice as long as the counterpart of $B$ by the perceivers phenomenal twin on Doubled Earth. The ratio between all magnitudes is the same on Earth as on Doubled Earth.

What I have said so far is presumably not only true with respect to Doubled Earth. We may suppose not only that Doubled Earth articulates a possible Twin Earth Scenario, but also that Heavy Earth articulates a possible Twin Earth Scenario. Heavy Earth is similar to Earth in all relevant respects except in two respects: All objects are twice as heavy on Heavy Earth as on Earth and our phenomenal twins on Heavy Earth are so much stronger than us that they lift objects which are twice as heavy as we do given the same efforts. My twin on Heavy Earth has the same experiences as I have, except that his experience of weight reliably tracks objects that are twice as heavy as my corresponding experiences. We may without much further ado suppose that we represent different weights.

Is Heavy Earth a possible scenario? I see no reason why it should not be. We can obviously draw the same conclusions from the case of Heavy Earth as we did from Doubled Earth. We can refer to Doubled Earth and Heavy Earth as examples of Metrical Earths, where the ratio between magnitudes remain the same but where the metrics is changed such that the ratio between a magnitude on one of the metrical earths and its counterpart on Earth is altered. If the above considerations are correct, metrics are in general twin-earthable.

Metrical Earths are twin-earthable because the relationship between the type of magnitude that functions as the vehicle of representation and the type of magnitude that is represented is contingent. The magnitude that is doing the representing is of a different kind than the magnitude that is represented. So there is nothing that prevents the representing magnitudes from representing other magnitudes than they actually do on Earth as long as there is no violation of dimensionality and relationality. We could quite easily conceive of a possible world in which phenomenal weights were the 
vehicles for representing volumes rather than weights since both weight and volume are one-dimensional properties.

We can contrast Metrical Earths with Logarithmic Earths. Logarithmic Earths are such that the ratio between the represented magnitudes are not isomorphic with respect to our worlds. Thus we may suppose that there is a Logarithmic Earth such that the vehicle for representing $1 \mathrm{~m}$ is the same on Earth as on Logarithmic Earth, but where the vehicle for representing $2 \mathrm{~m}$ on Earth, represent $4 \mathrm{~m}$ on Logarithmic Earth, the vehicle for representing $3 \mathrm{~m}$ on Earth, represent $9 \mathrm{~m}$ on Logarithmic Earth and the vehicle for representing $4 \mathrm{~m}$ on Earth, represent $16 \mathrm{~m}$ on Logarithmic Earth, and so on and so forth.

Is Logarithmic Earth a successful Twin Earth scenario? It seems to me that this is not the case. The reason is that we can perceive not only that there is a distance between two points in space, but also that some points lies halfway between these points. Thus, for example, if $\mathrm{B}$ is the point that lies half the way between $\mathrm{A}$ and $\mathrm{C}$, then the distance $\mathrm{AC}$ will be identical to the sum of the distances $\mathrm{AB}$ and $\mathrm{BC}$.

Let us assume that $\mathrm{AB}$ and $\mathrm{BC}$ represent $2 \mathrm{~m}$ on Earth, and the vehicle for representing $\mathrm{AC}$ represents $4 \mathrm{~m}$. What would these vehicles represent on Logarithmic Earth? The vehicle for representing $4 \mathrm{~m}$ on Earth, will by assumption represent $16 \mathrm{~m}$ on Logarithmic Earth. And the vehicle for representing $2 \mathrm{~m}$ on Earth will by assumption represent $4 \mathrm{~m}$ on Earth. But this will clearly not work. For $\mathrm{AC}$ is the fusion of $\mathrm{AB}$ and $\mathrm{BC}$, and since $\mathrm{AB}$ and $\mathrm{BC}$ represent $4 \mathrm{~m}$, the fusion of $\mathrm{AC}$ must represent $8 \mathrm{~m}$. But this runs counter to the initial assumption that $\mathrm{AC}$ would represent $16 \mathrm{~m}$.

Let us consider a different scenario, which we may call Expanding Earth. Expanding Earth is populated by our phenomenal twins, but it is different from Earth in the sense that for some strange reason the size of the Earth is continuously expanding in all three dimensions - it doubles its size every month. The shapes of the objects are the same as on Earth. It is only distances, areas and volumes that are continuously expanding.

We may assume that the inhabitants on Expanding Earth never detects that their world is continuously expanding. But what are the distances in phenomenal space on Expanding Earth representing? Could a case not be made that distances in phenomenal space would in this case represent logarithmically?

My answer would be negative. Distances in phenomenal space would in this case not represent anything since the "scale" of the world is continuously changing. Distances in phenomenal space would be correlated with different distances at different points in time. They would not reliably track any determinate distance. If A and B are determinate positions in phenomenal space that represent points on Expanding Earth, then the distance $\mathrm{AB}$ in phenomenal space will be correlated with different distances at different times.

However, phenomenal shapes on Expanding Earth would still represent shapes. So the representation of shapes of objects and configurations of objects would be the same on Earth as on Expanding Earth. This means that our phenomenal twins have an impoverished content in comparison to us-we represent shapes and distances. They will only represent shapes. Of course, in virtue of representing shapes, they will also be able to represent ratios between distances at a given point in time. So if they perceive a square, they will represent the lengths of the sides as being identical to each other at that point in time. But they will not represent the distance of the lengths. 
This is not to deny that some phenomenal magnitudes could be twin-earthable in this way. Perhaps phenomenal weight could represent exponentially on Logarithmic Earth. Spatial distance is different from other magnitudes in the sense that distances can be perceived as being the sums of its parts. But it is far from obvious that we could, for example, perceive weights to be the sum of its parts in an analogous way. And then a case could be made that phenomenal weights could represent in an exponential way.

Be that as it may. What matters in this context is that phenomenal distances cannot represent in the way suggested by the logarithmic earth scenarios. If we assume otherwise, we run into contradictions.

\section{Points in phenomenal space}

Phenomenal space is, as we have seen, a geometrical entity. It consists of a manifold of points that are related to each other in three dimensions. What could these points possibly represent? I shall suggest that proposition (iii) is correct and that points are only able to represent points in a space.

(iii) Points in phenomenal space can only represent points in a space.

As I stressed when introducing the notion of points, points can together constitute regions, and nothing of any importance hinges on conceiving of them as lacking extension in all dimensions. The proposition should be read as entailing that positions in phenomenal space in general can only represent positions in a space.

Before we proceed, we should note that the relevant points populate threedimensional structures and that they bear distance-relations to each other. That sets some constraints on what points could possibly represent, since, per relationality, the relationships that the points in phenomenal space bear to each other are also vehicles of representation. Consequently, any successful Twin Earth scenario would have to claim that the points are reliably tracking entities that are located in a three-dimensional structure of some kind and that can bear distance-relations to the other points in the structure.

This constraint effectively narrows the options for what points can reliably track. They must reliably track entities which are located in a three-dimensional structure. As far as I can see, that leaves us with only two options. The first option is that points can reliably track points in a space. We already know that this is the case, since this is what points in phenomenal space reliably track on Earth.

The second option would be to hold that points in phenomenal space can reliably track points in a property space of some kind. We have seen that properties could metaphorically be described as forming property-spaces of various dimensions. Does not this show that points in phenomenal space could reliably track values in property space rather than points in space?

Actually, it shows no such thing. What the example demonstrates is merely that property-spaces could be represented in a multidimensional and analogue form. This is perhaps best seen if we ponder what the relata of distances in property space are.

Let us assume that blue is located between red and green in colour space and that it is necessarily the case that blue and red resemble each other more than green and red. This follows from the very fact that the distance between blue and red is shorter than 
the distance between green and red. For the distance between two shades in colour space is not a contingent matter; it is grounded in the nature of the shades themselves. The relata of distances in property spaces are properties.

In real spaces however, the relata of distances are points or sets of points that compose regions. But there are no intrinsic differences between points. Positions in space can obviously be occupied by different types of entities. But they themselves (or the points that compose them) do not differ from each other. They are all type-identical. All points in property space differ from each other. All points in real spaces-whether physical or phenomenal-however are type-identical. They are not intrinsically different. A fortiori, points in phenomenal space cannot reliably track different types of entities. They must all reliably track the same types of entities. So they cannot reliably track properties in a property-space, for these all differ from each other.

There is a further important difference between points in spaces and properties in property-spaces. A three-dimensional spatial structure may obviously be used to represent a property space with three dimensions. But if we conceive of the structure itself in abstraction from the entities that occupy locations in it, it is merely a geometrical entity of (in this case) three dimensions. This entity can be used to represent resemblancerelations between different properties (for example in colour-space) when the locations in the structure are occupied by vehicles of representation (for example colours or various numerical values). In itself however, it is merely a structure with positions that may or may not be occupied by various entities. Thus, for example, colour-spaces are usually represented by three-dimensional entities where each point in the structure is occupied by a colour that represents itself. Points can in other words be occupied by entities with which they are not type-identical. Properties however cannot be occupied by any entity. But they can be the entity that occupies points in a space.

Points in property-spaces consequently differ from points in phenomenal spaces in two senses. No pair of points in a property space are type-identical. All points in phenomenal space are type-identical. No point in a property space can be occupied by a different kind of entity. All points in phenomenal space can be occupied by a different kind of entity. Points in space are consequently unable to represent any properties. Hence, if they reliably track anything, they reliably track points in spaces.

\section{Occupation}

Positions in space have a further characteristic that is important for our purpose. We have seen that a position in space can function as a location that is occupied by other types of entities than a position in a space. Points in space are in other words positions in a structure which may or may not be occupied by other entities. In this section I shall defend proposition (iv).

(iv) The occupation-relation in phenomenal space can only represent that a position in a space is occupied by something.

If a position in phenomenal space is occupied by an entity, then the relation between the position and the entity located there can in other words only represent an occupationrelation. 
It is trivially true that positions in physical space can be occupied by ordinary physical objects. But it is important to point out that positions in phenomenal space can also be occupied by objects, albeit not physical objects. Thus, for example, a position in phenomenal space may be occupied by a vehicle representing an object of some kind. If you perceive a ball, there is a phenomenal entity that occupies a position in your phenomenal space and that functions as the vehicle for perceiving the ball.

When one object occupies a position it has the same spatial position as that position and it "inherits" its spatial relations. So if the position is located $2 \mathrm{~m}$ from another position, then the object occupying the position is also located $2 \mathrm{~m}$ from that position. The entities that occupy positions are in other words themselves able to have the same kind of spatial relations as the positions they occupy.

The relation that a position in phenomenal space bears to what-if anything-is occupying that position is consequently itself a vehicle of representation. Positions in phenomenal space are the relata of two distinct types of relations that functions as vehicles of representation on Earth: Spatial distance-relations and occupation-relations, where the latter relation is to an entity that enters into the same distance-relations as the points themselves.

Now, it is hard to see how the occupation-relation that holds between a position and a vehicle for representing an object in phenomenal space could reliably track some other entity than an occupation-relation between a position and an object occupying that position in a space. For the entity that occupies a position in phenomenal space is not identical to a position in phenomenal space, yet it nevertheless bears the same spatial relationships to other positions as the position it is occupying does.

It is quite clear that the kind of entities that can occupy positions in phenomenal space are not themselves positions in space. Yet they must be able to bear all the spatial relations that positions in space bear and, so to speak, coincide in space with such a position. This fact, however, effectively rules out that the occupation relation in phenomenal space could be the vehicle for representing anything but positions being occupied by objects of some kind in space.

In order to see why the occupation-relation must be the vehicle for representing occupation-relations we might ponder a Twin Earth scenario in which it was not the vehicle for representing occupation-relations. In such a case the relata of the occupation-relations in phenomenal space would be the vehicles of representing other entities than they actually do in our world.

The first alternative would be that positions in the phenomenal space were not the vehicles of representation for positions in space. We have however seen that positions in phenomenal space cannot represent anything but positions in a space. Consequently, this alternative does not give us a successful Twin Earth scenario since the representational content on our Twin Earth would be impoverished.

The alternative would be to hold that the other relatum of the occupationrelation - the entity that is occupying the position in phenomenal space and functions as the vehicle for representing ordinary material objects on Earth-would have to be the vehicle for representing other entities than those that occupy spatial positions. This, however, does not work either. For if the occupying entities in phenomenal space were not the vehicles for representing entities that occupy spatial positions, then the relations that these entities bear to positions in phenomenal space would not represent 
anything. But that would once again leave us with an impoverished representational content.

I conclude that the occupation relation in phenomenal space must be the vehicle for representing occupation-relations in a space.

Here, however, we encounter a possible objection. Unless you are a reductive physicalist with respect to consciousness, the kind of entities that occupy locations in physical space are presumably different than the kind of entities that occupy locations in phenomenal space. Phenomenal space and physical space are in other words different kinds of entities. They are isomorphic in the sense that they have the same kind of geometrical structure, but they are nevertheless different kinds of entities. So could we not conceive of a world where phenomenal space reliably tracked some non-physical space which nevertheless had the same kind of geometric structure as phenomenal and physical space?

I see no reason to deny this consequence. So, for example, if spaces could supervene on computational facts, it is possible that a phenomenal twin of ours who was a brain in a vat could represent the structure of a computational space. I think that this offers a way out for those who wish to argue that brains in vats have as rich a representational content as we do. Nevertheless, any such claim rests on the unproven assumption that there could be such a thing as a computational space.

We should note that it is not clear how many different kinds of spaces there are, or indeed if there even are any more kinds than phenomenal and physical space. But if there are other kinds of spaces that could be systematically related to phenomenal space, then the latter could conceivably represent other kinds of spaces than physical space.

Nevertheless, phenomenal space could not reliably track any entity that was not itself a geometrical structure and that contained points which were connected by distances. My claim is that phenomenal space can only represent entities with a spatial structure, but not that it can only represent physical spaces. So even though brains in vats might be able to represent non-physical spaces, such a Twin Earth scenario would not be a successful Twin Earth scenario in the sense that it showed that the vehicles for representing geometrical entities on Earth, could represent other kinds of entities for a brain in a vat. The brain in a vat would represent the same geometrical entities that we do, but the kinds of objects represented as occupying the positions in the represented space would be different for the brain in a vat.

\section{Concluding words}

I have argued that while there are successful Twin Earth scenarios, there are also constraints that prevent some Twin Earth scenarios. I have illustrated these constraints by pointing at four structural features of phenomenal space. Phenomenal space has three dimensions. It consists of points. These points can be occupied. Points in phenomenal space are related by a distance. We can now see that the four claims (i)-(iv) entails that these structural features can only represent entities with which they are type-identical. Three-dimensional phenomenal space can only represent three spatial dimensions. The ratio between distances in phenomenal space can only represent the 
same ratios between distances in space. The points in phenomenal space can only represent points in a space. And the occupation-relation that holds between points in phenomenal space and the entities that occupy them can only represent that a point in a space is occupied by some entity.

The argument given so far does not in itself demonstrate that phenomenal space is intrinsically intentional. It could still be suggested that phenomenal space is only representational if it bears a tracking-relation of some kind to a spatial structure. Nevertheless, since the argument demonstrates that the structure of phenomenal space is not amenable to Twin Earth scenarios, the argument could be used as a first step in an argument to the effect that there are some vehicles of representations which are intrinsically representational.

I will finish the paper by briefly outlining some interesting consequences for an internalistic view of perceptual content, or at any rate ones that should be interesting if one is willing to accept David Chalmers notion of Edenic content. Chalmers (2006) has suggested that we should distinguish between Edenic and Fregean content, where Edenic content is a kind of content that presents the world as it is: "For the world to be exactly the way that my phenomenology seems to present it as being, the world would have to be an Edenic world in which these properties are instantiated." (Chalmers 2006: p. 66) Chalmers express Edenic content in terms of properties, but there is nothing that prevents the analysis from applying to structures as well.

Let us assume with Chalmers that phenomenal entities can form a kind of Edenic content. The essential feature of Edenic content is as I understand it that the vehicle of representation is type-identical with the object of representation in a veridical experience. The conditions of satisfaction for Edenic content requires that an entity that is type-identical to the vehicle of representation is suitably related to the perceiver. A consequence of this conception of Edenic content is that Edenic content is the same in all possible worlds. There is no possible scenario in which Edenic content can be made veridical by entities that are not type-identical to itself.

Chalmers contrasts Edenic content with Fregean content. Fregean content refers to the entity that is the reliable cause of the phenomenal property (Chalmers 2006: p. 59). The connection between the vehicle of representation and the object of representation is in this case contingent. The vehicle of representation is not itself a part of the conditions of satisfaction for Fregean content.

Chalmers suggests that Edenic content is never satisfied. The world is never exactly as it appears phenomenologically. There are plenty of convincing Twin Earth scenarios that shows that Chalmers is right about some phenomenal properties like colours. However, I would like to suggest that the structure of phenomenal space is a good candidate for a kind of Edenic content that actually can be satisfied.

If my argument is correct, the vehicles of representation for our four structural features are type-identical with what they represent. And the vehicles of representation cannot be made veridical by any other entity than those which are type-identical to themselves. Phenomenal space has a geometric structure, and it is veridical if the surrounding environment has the same structure, otherwise it is not veridical.

There is a further point that needs to be made. There is no possible scenario in which these structural features are carriers of Fregean content such that their conditions of satisfaction do not involve themselves. There is of course Doubled Earth, where the 
metrics is different than on Earth. But there is no scenario where phenomenal space is made veridical by an entity that lacks a geometrical structure or that have a geometrical structure with a different set of structural features than phenomenal space.

If the argumentation is correct, phenomenal space cannot represent any other entity but one that is type-identical to itself. And this content can, contrary to what Chalmers supposes, actually be satisfied. So if Chalmers is correct in holding that there is a kind of Edenic content, we have identified a vehicle of representation whose Edenic content can be satisfied, and which lacks a Fregean content. Given that Chalmers' distinction between Edenic and Fregean content is broadly correct, this entails that phenomenal space has an intrinsic content which can be satisfied, and which actually allows us to represent our environment in at least some contexts.

The conjunction of Chalmers' claim to the effect that phenomenal states and properties have a kind of Edenic content with my argument to the effect that the structure of phenomenal space only can represent a structure with which it is type-identical will in other words entail that there is a kind of internal phenomenal content which is intrinsically intentional, which can be satisfied by entities in the world, and which, when veridical, reveals the world as it is.

Acknowledgements Open access funding provided by Karlstad University. Thanks are due to Solveig Aasen, Alexander Almér, Rögnvaldur Ingthorsson, Kristoffer Sundberg and my two anonymous referees for valuable comments on a previous version of the paper.

Funding This work was funded by the Swedish Research Council, Grant 2017-02546.

Open Access This article is distributed under the terms of the Creative Commons Attribution 4.0 International License (http://creativecommons.org/licenses/by/4.0/), which permits unrestricted use, distribution, and reproduction in any medium, provided you give appropriate credit to the original author(s) and the source, provide a link to the Creative Commons license, and indicate if changes were made.

\section{References}

Almäng, J. (2017). An argument for shape internalism. Erkenntnis, 82, 819-836.

Beck, J. (2012). The generality constraint and the structure of thought. Mind, 121, 563-600.

Beck, J. (2019). Perception is analogue: The argument from Weber's law. Journal of Philosophy, 116, 319-349.

Block, N. (1990). Inverted earth. Philosophical Perspectives, 4, 53-79.

Burge, T. (2010). Origins of objectivity. Oxford: Clarendon Press.

Chalmers, D. (2006). Perception and the fall from Eden. In T. S. Gendler \& J. Hawthorne (Eds.), Perceptual experience (pp. 49-125). Oxford: Clarendon Press.

Chalmers, D. (2012). Constructing the world. Oxford: Oxford University Press.

Chalmers, D. (2018). Structuralism as a response to skepticism. The Journal of Philosophy, 115, 625-660.

Dainton, B. (2000). Stream of consciousness. Unity and continuity in conscious experience. London: Routledge.

Farkas, K. (2003). What is externalism? Philosophical Studies, 112, 187-208.

Farkas, K. (2008). The subject's point of view. Oxford: Oxford University Press.

Funkhouser, E. (2014). The logical structure of kinds. Oxford: Oxford University Press.

Gibson, J. J. (1950). The perception of the visual world. Cambridge, MA: The Riverside Press.

Hopp, W. (2013). No such look. Problems with the dual content theory. Phenomenology and the Cognitive Sciences, 12, 813-833.

Horgan, T., \& Graham, G. (2012). Phenomenal intentionality and content determinacy. In R. Schantz (Ed.), Prospects for meaning (pp. 321-344). Berlin: De Gruyter. 
Horgan, T., Tienson, J., \& Graham, G. (2004). Phenomenal intentionality and the brain in a vat. In R. Schantz (Ed.), The externalist challenge (pp. 297-317). Berlin: Walter de Gruyter.

Husserl, E. (1966). Analysen zur Passiven Synthesis. Aus Vorlesungs und Forschungsmanuskripten 1918-1926, hrsg. M. Fleischer, Haag: Martinus Nijhoff.

Jagnow, R. (2008). Disappearing appearances. On the enactive approach to spatial perceptual content. The Southern Journal of Philosophy, XLVI, 45-67.

Jagnow, R. (2012). Representationalism and the perspectival character of perceptual experience. Philosophical Studies, 157, 227-249.

Kriegel, U. (2011). The sources of intentionality. Oxford: Oxford University Press.

Kriegel, U. (2013). The phenomenal intentionality research program. In U. Kriegel (Ed.), Phenomenal intentionality (pp. 1-26). Oxford: Oxford University Press.

Lewis, D. (1984). Putnam's paradox. Australasian Journal of Philosophy, 62, 221-236.

Mac Cumhaill, C. (2015). Perceiving immaterial paths. Philosophy and Phenomenlogical Research, 90, 687-715.

Marr, D. (1982). Vision. A computational investigation into the human representation and processing of visual information. San Francisco: W.H. Freeman and Company.

Martin, M. (1993). Sense-modalities and spatial properties. In N. Eilan, R. McCarthy, \& B. Brewer (Eds.), Spatial representation. Problems in philosophy and psychology (pp. 206-218). Oxford: Oxford University Press.

Martin, M. (2004). The limits of self-awareness. Philosophical Studies, 120, 37-89.

Nerlich, G. (1994). The shape of space (2nd ed.). Cambridge: Cambridge University Press.

Nerlich, G. (2013). Einstein's genie. Spacetime out of the bottle. Montreal: Minkowski Institute Press.

Newman, M. H. A. (1928). Mr. Russell's "Causal theory of perception". Mind, 37, 137-148.

Peacocke, C. (1983). Sense and content. Oxford: Clarendon Press.

Putnam, H. (1973). Meaning and reference. The Journal of Philosophy, 70, 699-711.

Richardson, L. (2009). Seeing empty space. European Journal of Philosophy, 18, 227-243.

Siegel, S. (2010). The contents of visual experience. Oxford: Oxford University Press-.

Siewert, C. (1998). The significance of consciousness. Princeton: Princeton University Press.

Smith, A. D. (2002). The problem of perception. Cambridge, MA: Harvard University Press.

Smith, B. C. (1991). The owl and the electric encyclopedia. Artificial Intelligence, 47, 251-288.

Smith, D. W., \& McIntyre, R. (1982). Husserl and intentionality. A study of mind, meaning, and language. Dordrecht: Springer.

Snowdon, P. (2005). The formulation of disjunctivism. A response to fish. Proceedings of the Aristotelian Society, 105, 129-141.

Soteriou, M. (2011). The perception of absences, space and time. In J. Roessler, H. Lerman, \& N. Eilan (Eds.), Perception, causation and objectivity (pp. 181-206). Oxford: Oxford University Press.

Thompson, B. (2010). The spatial content of experience. Philosophy and Phenomenological Research, 81, $146-184$.

Tye, M. (1998). Inverted earth, swampman and representationism. Philosophical Perspectives, 12, 459-477.

Tye, M. (2002). Representationalism and the transparency of experience. Noûs, 36, 137-151.

Publisher's Note Springer Nature remains neutral with regard to jurisdictional claims in published maps and institutional affiliations. 